\title{
LA FLORA DE LAS BARRANCAS DEL COBRE, CHIHUAHUA, MÉXICO
}

\section{THE FLORA OF THE COPPER CANYON, CHIHUAHUA, MEXICO}

\author{
Toutcha Lebgue ${ }^{1}$, Manuel Sosa ${ }^{2}$ y Ricardo Soto ${ }^{3}$
}

\begin{abstract}
Resumen
Las Barrancas del Cobre, es una zona de grandes depresiones en relieve dentro de la Sierra Madre Occidental, esto le da una singularidad tanto en aspectos físicos como biológicos, por lo que su importancia es resaltada a nivel mundial. El objetivo del estudio fue obtener información de tipo taxonómico de las especies vegetales. El área de estudio tiene una superficie de 126653 hectáreas. Las Barrancas del Cobre, se extienden sobre los municipios de Batopilas y Urique, en la porción suroeste del Estado de Chihuahua, colindando con los estados de Sinaloa y Sonora. Su clima es de tipo subtropical con temperaturas altas de Marzo a Julio y lluvias de Junio a Septiembre. Las comunidades vegetales están constituidas principalmente por bosques tropicales caducifolios, cuyas especies dominantes son árboles y arbustos. El resultado del estudio el cual se realizó del año 1998 al 2003 consistió en un listado florístico que consta de 770 especies repartidas en 184 géneros y 121 familias; de estas últimas sobresalen: Poaceae con 134 especies, Asteraceae con 78, Fabaceae con 76, Solanaceae con 24, Euphorbiaceae con 20, Mimosaceae con 19, Malvaceae y Scrophulariaceae con 18 especies respectivamente. Se concluye que la biodiversidad de esta región es altamente rica y que la mayoría de las especies se concentró en el Bosque Tropical Caducifolio el cual representa la comunidad vegetal dominante en la región comparada con el Bosque de Encinos y de Coníferas encontrados en las partes más altas.
\end{abstract}

Palabras Claves: flora, Barrancas del Cobre, asociaciones vegetales, biodiversidad.

\begin{abstract}
Copper Canyon is a zone of deep geologic depressions within the Sierra Madre Occidental region. Its physical and biological features have singled it worldwide. The objective of this study was to obtain taxonomic information on the plant species. The study site has an area of 126653 hectares, extending over the Batopilas and Urique Municipalities, on the southwestern portion of the State of Chihuahua, bordering the States of Sonora and Sinaloa. A subtropical type climate dominates the Copper Canyon providing high temperatures from March to July and rainfall from June to September. The plant communities are mainly Tropical Deciduous Forests, with dominant plant species. The 5 year study $(1998-2003)$ resulted in a floristic check list of about 770 species, (184 genera and 121 families). The most dominant families in term of species were: Poaceae 134 species, Asteraceae 78 species, Fabaceae 76, Solanaceae 24, Euphorbiaceae 20, Mimosaceae 19, Malvaceae and Scrophulariaceae with 18 species each. It was concluded that the Copper Canyon biodiversity is highly rich and that the majority of the plant species were concentrated in the Tropical Deciduous Forest which is the dominant plant association compared to the Oak Forest and Coniferous Forest found in the higher areas of the region.
\end{abstract}

Key Words: flora, Copper Canyon, plant association, biodiversity.

\section{Introducción}

Las Barrancas del Cobre están dominadas por una Selva Baja Caducifolia (Bosque Tropical Caducifolio) caracterizada por un conjunto de bosques propios de regiones cálidas dominadas por especies arborescentes que pierden sus hojas durante la época seca del año, que por lo general oscila alrededor de seis meses (Rzedowski, 1983). Esta selva ocupa una franja muy pequeña pero importante para la biodiversidad para la entidad en la vertiente del Océano Pacífico, en el suroeste del Estado de Chihuahua, tocando porciones de Sonora y Sinaloa, predominando en las laderas y fondos de los barrancos con una latitud que va de 300 a $2000 \mathrm{msnm}$.
Las especies arbustivas y arbóreas se encuentran dentro de los géneros: Lysiloma, Ceiba, Ficus, Bursera, Celtis, Acacia, Senna, Caesalpinia, Haematoxylon, Quercus, Ipomoea, Tecoma, Prosopis, Mimosa, Fouquieria, Agave, Opuntia, Stenocereus, Pachycereus, entre otros, mientras que el estrato herbáceo está dominado por especies de Panicum, Paspalum, Eragrostis, Lassiacis, Muhlenbergia, Bouteloua, Setaria, Ambrosia, Bidens, Zinnia, Ipomoea, Cyperus, Euphorbia, Dalea, Desmodium, entre otros.

Florísticamente hablando, se han realizado más estudios en la Alta Tarahumara debido a su accesibilidad. Dentro de estos trabajos, se pueden 
citar: Vegetation of the Mountain Pima Village of Nabogame, Chihuahua, realizado por Laferriere (1994) reportando algunas 600 especies de plantas vasculares, las cuales son dominadas por especies de los géneros Pinus y Quercus generando así los Bosques de Pino o Pino-Encino.

Un estudio similar fue llevado a cabo por Spellenberg et al. (1996) en el cual se reportan más de 800 especies de plantas que incluyen partes de las barrancas (Barrancas de Candameña).

Lebgue \& Valerio (1991) citan los géneros Bromus, Agrostis, Muhlenbergia, Elytrigia, Aegopogon, Aristida, Schizachyrium, Bouteloua, entre otros, como los más dominantes dentro de la familia de las gramíneas, constituyendo especies que crecen debajo de los árboles de pinos y encinos, ocupando las zonas de aclareos en los bosques.

Aunque no directamente relacionados con las barrancas de Chihuahua, los estudios realizados por Felger et al, (2001) hacen una clasificación de los tipos mayores de vegetación que dominan en la zona incluyendo el Bosque Tropical Caducifolio, como una vegetación típica de las barrancas.

El precursor de los estudios en el Estado de Sonora fue Howard S. Gentry quien realizó una investigación exhaustiva de 1933 hasta 1951, sus resultados fueron publicados por Martín et al, (1998) donde reconoce la presencia de tres tipos de vegetación en las barrancas: Vegetación Ribereña, Bosque Tropical Caducifolio y Bosque de Encino.

Siendo el estado más grande de México, la Flora de Chihuahua, aún se encuentra en la fase de compilación de datos. Existen varios estudios ya terminados, pero la mayoría de ellos están dirigidos hacia los aspectos taxonómicos, y pocos tratan los componentes ecológicos de la vegetación de la entidad. Asimismo, la realización de un trabajo tanto taxonómico como ecológico, aunque dirigido hacia una sola región, como las Barrancas del Cobre, es de mucho interés ya que aporta información necesaria para hacer de la Flora de Chihuahua un elemento más estudiado, y se genere un documento que será de mucha utilidad para la comunidad científica, tanto de esta misma entidad federativa como para el país en general.

El objetivo principal fue de inventariar las especies de las plantas vasculares de las Barrancas del Cobre.

\section{Materiales y Métodos}

Localización. La Baja Tarahumara o región de las Barrancas del Cobre, con una superficie de 126653 hectáreas, está ubicada entre las coordenadas geográficas 27:14:42 Lat. N. Máxima, 26:42:44 Lat. N. Mínima y 108:02:01 Long. W. Máxima, 107:32:56: Long. W. Mínima. Se ubica físicamente en la parte suroeste del Estado de Chihuahua, colindando con los Estados de Sonora y Sinaloa.
Clima. La región está dominada por un clima semiseco muy cálido y cálido de tipo $\mathrm{A}(\mathrm{c})$ de acuerdo con Koppen modificado por García (1974 ), el cual genera una vegetación que exhibe características tropicales, en el sentido de que sus especies botánicas están adaptadas a regímenes de temperaturas altas, con ausencia de temperaturas cercanas a cero grado centígrado durante casi todo el año, y además estas especies pierden sus hojas durante un período del año, generando así lo que se conoce como Bosque Tropical Caducifolio. La precipitación oscila entre los 1200 $\mathrm{mm}$ en las partes altas, a los $2000 \mathrm{msnm}$ (Pueblo de Kirare) y más, y los $550 \mathrm{~mm}$ en las partes bajas, a los $300 \mathrm{msnm}$ (Pueblo de Tubares). La temperatura varia de $10.5^{\circ} \mathrm{C}$ (mínima) y $29^{\circ} \mathrm{C}$ (máxima) en los Bosques de Pino-Encino a los $2000 \mathrm{msnm}$ hasta $17^{\circ} \mathrm{C}$ (mínima) y $39^{\circ} \mathrm{C}$ (máxima) en los Bosques Tropicales Caducifolios, a los $300 \mathrm{msnm}$ o menos, con promedios anuales de $20^{\circ} \mathrm{C}$ y $28^{\circ} \mathrm{C}$ respectivamente.

Suelos. Los suelos de la zona de estudio se dividen en dos categorías: el feozem haplico y el cambisol cromico dominan en las partes bajas de las barrancas en donde prevalece un clima más calido, mientras que el litosol y el rigosol eutrico son encontrados en las partes más altas donde dominan los Bosques de Pino-Encinos. El feozem haplico es un suelo caracterizado en tener una superficie oscura, suave y rica en materia organica y nutrientes. El cambisol cromico es un suelo joven, poco desarrollado y se caracteriza en tener una capa de terrones que presentan un cambio con respecto al tipo de roca subyacente con alguna acumulación de arcilla, calcio, etc. El litosol es un suelo sin desarrollo con profundidad menor de $10 \mathrm{~cm}$, y con características muy variables según el material que lo forma. El rigosol eutrico es un suelo de capas distintas, de color claro y se parece a las rocas que lo forman. En forma general, todos estos suelos son de formación in-situ, delgados, poco profundos, con muy poca materia orgánica, de textura pedregosa, debido a que casi todas las partículas están arrastradas por las aguas resultando de las pendientes pronunciadas de las formaciones geológicas en la región (SPP, 1981).

Geología. Las Barrancas del Cobre se encuentran ubicadas en la parte norcentral de la Provincia Fisiográfica de la Sierra Madre Occidental, en la porción suroeste del Estado de Chihuahua donde se colinda con los Estados de Sonora y Sinaloa.

Esta Provincia se extiende por el noroeste desde la línea internacional con los Estados Unidos (Arizona Sonora) hacia el interior de México con una dirección sureste, abarcando porciones de Sonora, Chihuahua, Durango, Sinaloa y parte del Estado de Jalisco, con una longitud de $1240 \mathrm{~km}$ y un promedio de $200 \mathrm{~km}$ de ancho. Colinda en el noreste con la Provincia Fisiográfica de Cuencas y Sierras Paralelas; en el sureste y sur con la Meseta Central y el Eje Volcánico 
Transmexicano; en el oeste y suroeste con la Planicie Costera del Golfo de Baja California y del Pacífico.

La Provincia Fisiográfica de la Sierra Madre Occidental se subdivide en tres subprovincias: las subprovincias de las Cuencas Altas y Sierra, las subprovincias del Altiplano y las subprovincias de Barrancas de Este y Oeste respectivamente.

$\mathrm{El}$ área de estudio se ubica en las subprovincias de las Barrancas de Este y Oeste, con un desnivel de casi $2000 \mathrm{~m}$. Esta zona está caracterizada por laderas muy escarpadas en sus partes superiores. Las mesetas que ahí se formaron están constituidas por rocas piroclásticas y en menor proporción flujos de lava y conglomerados. El patrón de drenaje es dendrítico rectangular y todas las corrientes se encuentran en la etapa juvenil.

La parte inferior de las Barrancas del Cobre está constituida principalmente por rocas andesíticas, las cuales son erosionadas por numerosos arroyos tributarios de los dos ríos principales: río Batopilas y río Urique. Los cauces principales de estos dos ríos y todos los arroyos secundarios disectan la región produciendo una topografía sumamente abrupta, llena de cañones o barrancos profundos. Entre las barrancas más importantes que aquí encontramos (entre paréntesis se anota su profundidad en metros) podemos citar a la de Urique (1 $879 \mathrm{~m}$ ), Sinforosa (1 $830 \mathrm{~m})$, Batopilas (1 $800 \mathrm{~m})$, lCandameña $(1750 \mathrm{~m})$, Río Mayo (1 680 m), Huápoca (1 620 m), Chínipas (1 $600 \mathrm{~m})$, Septentrión $(1600 \mathrm{~m})$ y Oteros $(1520 \mathrm{~m}), \mathrm{y}$ esto sólo por citar las más hondas, las que rebasan al famoso Cañón del Colorado. El sistema debe su nombre a la Barranca del Cobre, un ramal de la Barranca de Urique que alcanza los 1300 metros de hondura, y que se hizo notorio por sus minas de cobre".

Hidrología. El Sistema Hidrológico de las barrancas pertenece a la Región Hidrológica 10 (RH10-SINALOA) caracterizada por las corrientes de agua que nacen en la vertiente oeste de la Sierra Madre Occidental y corren hacia el Oeste para verter sus aguas en el Océano Pacífico pasando por el Golfo de California (INEGI, 1999). La RH 10 es una de las grandes regiones hidrológicas del país, abarcando los municipios de: Guadalupe y Calvo, Guachochi, Batopilas, Urique, Chinipas y Morelos.

La RH 10 comprende tres cuencas: Cuenca del Río Fuerte, Cuenca del Río Sinaloa y Cuenca del Río Culiacán. Todas las aguas de las Barrancas del Cobre forman parte de la Cuenca del Río Fuerte, la cual está constituida por 7 afluentes: Río Verde, Río San Miguel, Río Batopilas, Río Urique, Río Septentrión, Río Chinipas-Otero, y Río Fuerte. Mediante la Cuenca del Río Fuerte, el Estado de Chihuahua suministra al Estado de Sinaloa un volumen de agua equivalente a 3 473.73 millones de $\mathrm{m}^{3}$ equivalente a una lamina de agua de $140.96 \mathrm{~mm}$.
La zona de estudio se extiende sobre dos subcuencas: la subcuenca del río Batopilas y la del río Urique. El río Batopilas nace al Sur del Rancho San Miguel recorre en dirección suroeste, pasando por Basigochi, La Bufa y el pueblo de Batopilas, una distancia de $140 \mathrm{~km}$ para unirse luego con el Río San Miguel. Mientras que el río Urique tiene una longitud de $227 \mathrm{~km}$ y desde su nacimiento en la región de San Juanito, recorre la sierra pasando por el pueblo de Norogachi, Umirá, después entra a la región de las barrancas uniéndose luego con el río San Miguel pasando por el pueblo de Urique. Ambos ríos son caracterizados por corriente permanente de agua, ya que gracias a sus numerosos tributarios, sus aguas corren durante todo el año, aunque el nivel del caudal baja un poco durante el período sin lluvias.

Vegetación. La vegetación de las barrancas va variando desde los Bosques de Encinos encontrados en las partes más altas (más de $2000 \mathrm{msnm}$ ), donde dominan las especies de árboles como Quercus arizonica, $Q$. toumeyi, $Q$. oblongifolia, Pinus leiophylla, P. engelmannii, entre otras, pasando por los matorrales de huizaches (Acacia farnesiana) a los 250 o $300 \mathrm{msnm}$; asociados con especies tales como Acacia cochliacantha, Ipomoea chilopsidis, Randia thurberi, Rhus tepetate, Quercus chihuahuensis, entre otras y los Bosques de mauto (Lysiloma divaricatum) formado por las asociaciones de especies de árboles y arbustos adaptados en los ambientes con temperaturas altas como: Lysiloma wootonii, Ceiba pentandra, Guazuma ulmifolia, Senna atomaria, Ipomoea arborescens, Caesalpinia platyloba, Pachycereus pecten-arboriginum, Stenocereus thurberi, Bursera lancifolia, B. pinicillata, B. grandiflora, Cordia sonorae, Buddleja marrubifolia, Quercus albocincta, Q. tuberculata, Prosopis palmeri, P. articulata, Chloroleucon mangense, Fouquieria mcdougalii, entre muchas otras; para llegar hasta los Bosques Ribereños de higueras (Ficus petiolaris, F. pertusa, F. conitifolia), de guamuchil (Pithecellobium dulce) y de tempisque (Sideroxylon tepicense) formados sobre las orillas de los ríos Batopilas y Urique, juntos con especies de Brongniartia alamosana, Coccoloba goldmanii, Tabenuia chrysantha, T. Impetiginosa, Platimiscium trifoliolatum, Vitex mollis, $V$. pyramidata, entre otras.

La gran mayoría de estas especies, tiene un comportamiento típico de un clima subtropical, permanecen sin hojas por un periodo de casi seis meses durante el año (Rzedowski, 1983), excepto las especies de los bosques de las partes más altas. Asimismo, la vegetación se manifiesta en su forma exuberante durante la época de lluvias, apreciable en los meses de Julio hasta Enero o Febrero.

\section{Metodología}

Materiales y fuentes de datos. Para la realización de este estudio, se utilizó un sistema de posicionamiento 
global (GPS), el cual se utilizó para georeferenciar cada especie de plantas recolectadas y un mapa topográfico a escala 1:250 000 para ubicar los sitios de colecta de especimenes.

Colecta de especimenes de plantas. Se establecieron físicamente 18 grandes sitios de colecta en el área de estudio: 8 de ellos en la barranca de Batopilas y 10 en la de Urique. Dichos sitios fueron escogidos por a) el gradiente altitudinal, b) el tipo de vegetación dominante y c) su accesibilidad.

Los sitios de colecta de la Barranca de Batopilas son los siguientes:

Sitio 1: $4 \mathrm{~km}$ norte de Kirare. Altitud: $2100-2200$ msnm. Tipo de vegetación: Bosque de pino-encino. Coordenadas: 107:32:44 / 27:11:16

Sitio 2: Pueblo de Kirare. Altitud: $2000-2100$ msnm. Tipo de vegetación: Bosque de Pino-encino. Coordenadas: 107:33:45 / 27:09:44.

Sitio 3: $3 \mathrm{~km}$ sur de Kirare. Altitud: $1800-1900$ msnm. Tipo de vegetación: Bosque de encinos. Coordenadas: 107:34:18 / 27:08:36.

Sitio 4: La casita. Altitud: $1500-1650$ msnm. Tipo de vegetación: Bosque tropical caducifolio. Coordenadas: 107:33:55 / 27:07:55

Sitio 5: Bacuseachi. Altitud: $1000-1100 \mathrm{msnm}$. Tipo de vegetación: Bosque tropical caducifolio. Coordenadas: 107:37:10 / 27:07:01

Sitio 6: Arroyo de Santiago. Altitud: $500-650 \mathrm{msnm}$. Tipo de vegetación: Bosque tropical caducifolio. Coordenadas: 107:41:54 / 27:04:13

Sitio 7: Pueblo de Batopilas. Altitud: 450 - 500 msnm. Tipo de vegetación:

Bosque tropical caducifolio. Coordenadas: 107:44:17 / 27:01:38

Sitio 8: Pueblo de Satevo. Altitud: 300 - $500 \mathrm{msnm}$. Tipo de vegetación: Bosque tropical caducifolio. Coordenadas: 107:42:48 / 26: 59:01

Los sitios de colecta de la Barranca de Urique son los siguientes:

Sitio 1: Pueblo de Guadalupe. Altitud: 450 - 550 msnm. Tipo de Vegetación: Bosque tropical caducifolio. Coordenadas: 107:52:45 / 27:14:43

Sitio 2: Pueblo La Laja de Rodríguez. Altitud: 450 $500 \mathrm{msnm}$. Tipo de vegetación: bosque tropical caducifolio. Coordenadas: 107:53:00 / 27:09:29

Sitio 3: Pueblo de Urique. Altitud: 400 - $500 \mathrm{msnm}$. Tipo de vegetación: Bosque tropical caducifolio. Coordenadas: 107:54:32 / 27:13:16

Sitio 4: La Pinosa. Altitud: $1300-1450$ msnm. Tipo de vegetación: Bosque tropical caducifolio. Coordenadas: 107:56:07 / 27:13:17

Sitio 5: Camino a La Lajas de Rodríguez. Altitud: 500 - $550 \mathrm{msnm}$. Tipo de vegetación: Bosque tropical caducifolio. Coordenadas: 107:53:06 / 27:10:06

Sitio 6: Divisadero Urique. Altitud: $2000-2100$ msnm. Tipo de vegetación: Bosque pino-encino. Coordenadas: 107:56:25 / 27:14:07
Sitio 7: Intersección Camino a Guadalupe con el río Urique. Altitud: 550 a 650 msnm. Tipo de vegetación: Bosque tropical caducifolio. Coordenadas: 107:54:12 / 27:13:29

Sitio 8: Área del Sauzal. Altitud: 300 - 350 msnm. Tipo de vegetación: Bosque tropical caducifolio. Coordenadas: 107:52:52 / 26:59:28

Sitio 9: Área del Sauzal: Altitud: 700 - $850 \mathrm{msnm}$. Tipo de vegetación: Bosque tropical caducifolio. Coordenadas: 107:57:20 / 26:57:47

Sitio de colecta 10: Pueblo el Triguillo. Altitud: 1900 - $2000 \mathrm{msnm}$. Tipo de vegetación: Bosque de pinoencino. Coordenadas: 107:59:19/27:05:18.

La recolecta de los especimenes se realizó desde el mes de Noviembre de 1998 hasta el mes de Septiembre de 2001. Todos los sitios fueron visitados por lo menos una vez. Los sitios de Kirare, la Casita, Arroyo de Santiago y Satevó localizados en la Barranca de Batopilas así como los localizados en el Divisadero, La Lajas de Rodríguez, Guadalupe y el Sauzal, del lado de la Barranca de Urique, fueron visitados de 3 a 5 veces durante los 3 años del estudio. Sin embargo, debido a los factores ambientales que afectan la accesibilidad de la región, las colectas más fuertes se llevaron a cabo en los meses de Agosto, Septiembre, Octubre, Marzo y Abril de cada año. Las tres cuartas partes de la colecta total del proyecto se realizó en el año de 1999, en los meses de Agosto y Septiembre.

Como se puede apreciar, la colecta de las plantas se realizó desde las partes más altas (Barranca Batopilas: región de Kirare a $2100 \mathrm{msnm}$ y Barranca Urique: Divisadero también a $2100 \mathrm{msnm}$ ) hasta los $300 \mathrm{msnm}$ en el fondo de ambas.

Se usó el GPS para registrar la información espacial de cada especie de plantas recolectadas. Este registro se reporta tanto en coordenadas geográficas (Latitud y Longitud) como en UTM.

Identificación y manejo de los especimenes. Por carecer de información taxonómica de referencia fiel y digna sobre la vegetación de las Barrancas del Estado de Chihuahua, gran parte de los especimenes recolectados fueron enviados a expertos extranjeros y nacionales, especialistas en diferentes grupos taxonómicos para ser clasificados. Asimismo, se mandaron plantas a New Mexico State University, Las Cruces, NM, a CIIDIR, Durango, y al Instituto de Biología, México DF. El resto de las colectas fue identificado en la Universidad Autónoma de Chihuahua.

Se realizó un viaje especial a la Cd. de México a fin de consultar las colectas relacionadas con las barrancas de Chihuahua depositadas en el Herbario Nacional (MEXU). El resultado no fue satisfactorio ya que se encontraron muy pocos especimenes en el MEXU. Todos los especimenes recolectados fueron secados, fumigados, etiquetados y montados sobre cartulinas para ser depositados luego en el Herbario de 
la Universidad Autónoma de Chihuahua (CHIH), localizado en la Facultad de Zootecnia.

\section{Resultados y Discusión}

Las colectas de especimenes en el campo y la subsiguiente identificación de los mismos, revelaron la riqueza en biodiversidad de la zona de las Barrancas del Cobre, como se puede apreciar en los resultados obtenidos. La Tabla 1, reporta la relación de esta biodiversidad vegetal según las categorías de clasificación. Analizando la información del cuadro anterior, de las 121 familias, 28 de ellas son monotípicas y monogénericas o sea que sólo tienen un género con una sola especie, como es el caso de la Ebenaceae con Diospyros sonorae, Fouquieriaceae con Fouquieria Mcdougalii, Potamogetonaceae con $\mathrm{su}$ especie Potamogeton illinoensis, entre otras. Algunas familias tienen dos o más especies pero son monogénericas. Tal es el caso de la familia Chenopodiaceae con dos especies del único género Chenopodium; familia Bombacaceae tiene dos especies de su género Ceiba; familia Platanaceae tiene dos especies del género Platanus, familia Fagaceae con un solo género (Quercus) presente y 13 especies, entre otras. Sin embargo, la mayoría de las familias están constituidas con más de dos géneros o especies.

Tabla 1. La relación de la biodiversidad vegetal y taxa del Cañón del Cobre.

\begin{tabular}{lc}
\hline TAXA & Número de elementos \\
\hline Familias & 121 \\
Géneros & 184 \\
Especies & 766 \\
\hline
\end{tabular}

Tabla 2. La relación entre los tipos de vegetación y el número de especies.

\begin{tabular}{lc}
\hline Tipos de Vegetación & Número de especies \\
\hline Bosque de Coníferas & 92 \\
Bosque de Encinos & 76 \\
Bosque Tropical & 598 \\
Caducifolio & \\
\hline
\end{tabular}

Cuando se toma en cuenta la elasticidad de las familias, se encuentra lo siguiente: 1) la mayoría de las familias tienen especies confinadas exclusivamente en las partes medias o bajas de las barrancas como el caso de la Moracaeae con el género Ficus con todas sus especies, familia Bignoniaceae con el género Tabebuia y sus especies, familia Sterculiaceae con los géneros Guazuma y Waltheria y sus especies, entre otras; 2) algunas de ellas como las familias Fagaceae,
Fabaceae, Poaceae, Solanaceae, entre otras, tienen especies esparcidas tanto en las partes bajas, medias como altas; y 3) otras como las familias Pinaceae, Begoniaceae, Cupressaceae, Garryaceae, entre otras, prefieren las partes más altas donde las temperaturas son más templadas (rango de $10.5^{\circ}$ a $29^{\circ} \mathrm{C}$ ) y con mayor cantidad de precipitación (con promedio de $1200 \mathrm{~mm}$ ).

La Tabla 2, muestra la relación de especies con los tipos de vegetación mayores, observables en la colecta realizada desde las partes altas (más de $2000 \mathrm{msnm}$ ) hasta los fondos de los barrancos, a los $300 \mathrm{msnm}$. De acuerdo a este cuadro, al parecer existen 6 veces más especies de plantas en el Bosque Tropical Caducifoliio que en el Bosque de Coníferas o Bosque de Encinos; pero la realidad es que muchas de las especies encontradas en dicho bosque también crecen en los otros dos, pero por medio de los agentes de transporte como el agua, el viento o los animales, sus semillas son depositadas ahí y luego se adaptaron en las condiciones tropicales. La mayoría de ellas son hierbas o pastos perteneciendo a los géneros Muhlenbergia, Bouteloua, Bidens, Cyperus, Ipomoea, Commelina, Lobelia, Gnaphalium, Stevia, Zinnia, Eryngium, Euphorbia, Dalea, Phaseolus, Salvia, Sida, Panicum, Cenchrus, Eragrostis, Aristida, Digitaria, Mimulus, Solanum, entre otros. Este resultado coincide con el listado florístico del Parque Nacional Cascada de Basaseachi en donde se reportaron muchas de las mismas especies encontradas en las barrancas.

Tabla 3. La relación de las familias sobresaliente y el número de especies.

\begin{tabular}{lc}
\hline Familias sobresalientes & $\begin{array}{c}\text { Número de } \\
\text { especies }\end{array}$ \\
\hline POACEAE & 134 \\
ASTERACEAE & 78 \\
FABACEAE & 76 \\
SOLANACEAE & 24 \\
EUPHORBIACEAE & 20 \\
MIMOSACEAE & 19 \\
MALVACEAE & 18 \\
SCROPHULARIACEAE & 18 \\
\hline
\end{tabular}

La Tabla 3, señala las familias sobresalientes en cuanto a su diversidad en la zona de estudio y se puede observar que la flora de las Barrancas del Cobre, está dominada por las gramíneas o pastos (Poaceae) aportando casi la sexta parte de todas las especies encontradas. Existe una simple razón que explica esta dominancia de las gramíneas: son plantas polinizadas por el viento, sus semillas pueden ser transportadas tanto por el viento como por el agua, son 
las fuentes de alimentación de muchos animales y se adaptan fácilmente a los medios donde llegan. Después de las gramíneas siguen dos familias con un número similar de especies: 1) las compuestas (Asteraceae) que son plantas cosmopolitas, pero más adaptables a climas más templados y representadas por 78 especies, e igual que en el caso de los pastos, la mayoría de estas especies son hierbas cuyos diseminulos son acarreados desde arriba por los mismos agentes de transporte y depositados en las barrancas; y 2) las leguminosas (Fabaceae), plantas típicas de los trópicos ocupan el tercer lugar, pero a diferencia de las dos primeras, tienen más arbustos y árboles. Muchas de sus especies herbáceas llegaron también de las partes templadas. Combinando las tres familias de leguminosas (Fabaceae, Mimosaceae y Caesalpiniaceae), se suman 110 especies, haciendo de este grupo el segundo más grande en las barrancas. Las otras 4 familias (Solanaceae, Euphorbiaceae, Malvaceae, y Scrophulariaceae) son plantas muy comunes cuyas especies son encontradas en diversos tipos de habitats ocupando diferentes estratos vegetales aunque la mayoría de ellas son herbáceas.

Existen dos familias en la barrancas que se hacen notar, no tanto por el número de especies que tienen, sino por tener especies muy particulares de este ecosistema: la familia de los encinos (Fagaceae) y la familia de las cactáceas (Cactaceae). Los encinos son plantas muy comunes de la Sierra Madre occidental, con especies templadas que se asocian con especies de pinos ocupando un gradiente altitudinal desde los 1 500 hasta más de $3000 \mathrm{msnm}$. La misma familia se encuentra representada por especies tropicales (Quercus albocincta y Q. tuberculata) que se desarrollan desde los 1000 hasta los $300 \mathrm{msnm}$. Por otro lado, la familia de las cactáceas está reconocida como una de las familias típicas de los desiertos; sin embargo, se desarrolla perfectamente bien en los trópicos por medio de sus especies: Ferocactus pottsii (Biznaga), Pilosocereus alensis (Cardón barbón), Stenocereus thurberi (Pitaya), Pachycereus pectinarboriginum (Etcho), Opuntia robusta (Civiri), Mammillaria standleyi (Biznaguita), entre otras.

\section{Conclusiones}

En su contexto ecológico, la región de las Barrancas del Cobre representa un corredor importante para la biodiversidad en la Sierra Madre Occidental, caracterizada por la presencia de especies de plantas y animales que se encuentran en forma natural en los gradientes altitudinales y latitudinales diversos

Los resultados obtenidos fueron satisfactorios a pesar de las condiciones fisiográficamente difíciles encontradas en el medio. Sin embargo, por las mismas condiciones, aún existen lugares que no fueron explorados, así que el listado florístico arrojado en el presente estudio representa el $95 \%$ de la flora de la zona de estudio.

En cuanto al impacto ambiental, hoy en día, todas las barrancas son accesibles por vehículos, circulando por los diversos caminos construidos en la zona, ya sea para llegar a Batopilas, Urique, Chinipas, Moris, o a algunos otros asentamientos humanos. Existen diversos tipos de actividades que van desde la agricultura de subsistencia, de los indígenas mismos hasta las actividades mineras y turísticas en el área. A pesar de sus pendientes muy pronunciadas y con presencia de pocas mesetas planas, las barrancas son lugares algo llenos de humanos (los raramuris juntos con los mestizos), los cuales encontraron maneras de sobrevivir en ellas. En esta forma, son zonas altamente impactadas por el ser humano. Y tomando en cuenta todas estas actividades más lo delgado del horizonte $\mathrm{A}$ del suelo, las pendientes muy pronunciadas, y las condiciones climáticas cambiantes, se corre el riesgo de perder en un futuro no muy lejano algunos registros de plantas taxonomicamente importantes para la ciencia.

Se recomienda, gestiones políticas de parte del Gobierno del Estado ante la SEMARNAT para generar programas de conservación y protección a la biodiversidad de las Barrancas del Cobre.

Los indígenas así como los mestizos, habitantes de las barrancas utilizan muchas plantas como fuentes medicinales y muchas de ellas crecen en la entidad. Asimismo, se recomienda la realización de estudios de tipo etnobotánico para documentar estas plantas útiles en la vida del hombre.

\section{Literatura citada}

Felger, R.S., M.B. Johnson \& M.F. Wilson. 2001. The Trees of Sonora, Mexico. Oxford University Press. New York.

García, E. 1974. Modificaciones al sistema de clasificación climática de Koppen; UNAM. Instituto de Geografía.

INEGI . 1999. Estudio Hidrológico del Estado de Chihuahua. INEGI. Aguascalientes, Ags.

Laferriere, J.E. 1994. Vegetation and Flora of the Mountain Pima Village of Nabogame, Chihuahua, México.

Lebgue K., T. \& A. Valerio V. 1991. Gramineas de Chihuahua. Colección de Textos Universitarios. Universidad Autónoma de Chihuahua, Chihuahua, Chih. México.

Martin, Paul S., D. Yetman, M. Fisbein, P. Jenkins, T. R. Van Devender \& R.K. Wilson. 1998. Gentry's Río mayo Plants: The Tropical Deciduous Forest \& Environs of Northwest Mexico. The University of Arizona Press. Tucson, Az.

Rzedowski, J. 1983. Vegetación de México. Editorial Limusa. México DF.

Secretaria de Programación y de Presupuesto. 1981. Carta Edafológica. Dirección General de geografía del Territorio Nacional. México DF

Spellenberg, R., T. Lebgue K., \& R. I. Corral Díaz. 1996. A Specimen.based, Annotated Checklist of the Vascular Plants of Parque Nacional "Cascada de Basaseachi" and Adjacent Areas, Chihuahua, Mexico. Instituto de Biología. UNAM. México DF. 
${ }^{1}$ Universidad Autónoma de Chihuahua, Facultad de Zootecnia, Periférico Fco R. Almada Km. 1.Teléfono (614) 4340303 Fax 4 340345, tlebgue@uach.mx

${ }^{2}$ Universidad Autónoma de Chihuahua, Facultad de Zootecnia, Periférico Fco R. Almada Km. 1.Teléfono (614) 4340303 Fax 4340345 msosac@uach.mx

${ }^{3}$ Universidad Autónoma de Chihuahua, Facultad de Zootecnia, Periférico Fco R. Almada Km. 1.Teléfono (614) 4340303 Fax 4340345 rsoto@uach.mx 